\title{
A Constitutive Model of Metal Rubber for Hysteresis Characteristics Based on a Meso-Mechanical Method
}

\author{
Cao Fengli, Bai Hongbai, Li Dongwei, Ren Guoquan, Li Guozhang
}

Ordnance Engineering College, Shijiazhuang 050003, China

\begin{abstract}
Based on the typical mesoscopic structural characteristics of metal rubber, the mesoscopic physical mechanism was revealed through analyzing the spatial configuration and the contact mode of wire helixes for its compression deformation process. The mesoscopic structure unit based on the curved beam of variable length and the model of the contact interaction between curved beams were proposed. Combined with the distribution law of frictional contact points, a new constitutive model of metal rubber for hysteresis characteristics was established, which included its basic structure parameters such as the diameter and the elastic modulus of wire, the diameter of wire helix, and the relative density of metal rubber. The model could describe the restoring force curves of metal rubber in initial loading, unloading and repeated loading phases, and theoretically explained its elastic characteristics and dry friction damping characteristics of multipoint contact. To verify the theory model, a comparison was made between the theoretical results and the experimental results for metal rubber specimens with different relative densities. The results show that theoretic calculations are consistent with the experimental data, which will provides a theoretical basis for predicting the stiffness and damping of metal rubber and guiding its design.
\end{abstract}

Key words: metal rubber; mechanical properties; damping material; curved beam

Metal rubber is a new kind of dry friction damping material made of wire helixes. It combines the rubber-like elasticity with porosity, and possesses such properties as high damping, great ability to absorb the impact energy, obvious resistance to high and low temperatures, slow aging and so on. The material is widely used in many fields, especially in the sophisticated weapon equipment and aerospace fields, and presents a broad application prospect ${ }^{[1-3]}$.

In recent years, in-depth researches on the mechanical properties of metal rubber have been carried out; however, because of the complexity of its internal structure and nonlinear property, it becomes very difficult to analyze the mechanical properties of this kind of material. So far, there are two types of theoretical models on the basic mechanical properties of metal rubber, that is, the mathematical models and the physical models. According to the macro mechanical properties of metal rubber, the mathematical models are directly introduced ${ }^{[4-7]}$, which mainly include the double broken line, polynomial and mixed damping models. Because these models do not contain the basic structure parameters of metal rubber, they can not reasonably explain its physical performance of the stiffness and dry friction characteristics. Meanwhile, according to the structural characteristics of metal rubber, the approximate physical models were ascertained ${ }^{[8-11]}$, which mainly are the small beam, micro spring and porous material models. To some extent, the physical models can explain some mechanical properties of metal rubber, and also approximately describe its restoring force curves, but they can not predict its restoring force curves of predeformations, nor can they estimate its mechanical parameters.

It is clear that these models above are not perfect, and cannot provide a comprehensive description of mechanical properties of metal rubber. Therefore, it is very necessary to explore the physical mechanism of metal rubber further for its compression deformation process and to set up its high accuracy mechanical model. In the present paper, according to the main

Received date: January 25, 2015

Foundation item: Weapon Equipment Pre-research Foundation (51312060404); National Natural Science Foundation of China (51275525)

Corresponding author: Bai Hongbai, Ph. D., Professor, Department of Vehicle and Electric, Ordnance Engineering College, Shijiazhuang 050003, P. R. China, Tel: 0086-311-87994743,E-mail: bhbk12@sina.com

Copyright $($ C 2016, Northwest Institute for Nonferrous Metal Research. Published by Elsevier BV. All rights reserved. 
characteristics of mesoscopic deformation of metal rubber, the curved beam unit, whose length is related with the deformation history of metal rubber, was put forward, and combined with the distribution law of frictional contact points, a new constitutive model of metal rubber was established, so as to more fully reflect the mechanical properties of metal rubber.

\section{Hysteresis Characteristics of Metal Rubber}

The static hysteresis loops of metal rubber subjected to compressing loads are given in Fig.1, among which the large hysteresis loop is the loading and unloading process at the maximal displacement, and the small hysteresis loops are the loading and unloading process at arbitrary boundary point of the large hysteresis loop. The area of loading and unloading curves is power consumption of the friction within a cycle, and if the frictional influence is not taken into consideration, the loading curve and the unloading curve will overlap for the elastic force curve. Through the analysis above, metal rubber possesses mechanical properties of nonlinearity and asymmetry, which can be observed. Therefore it will be very difficult to describe its mechanical properties accurately.

For the large hysteresis loop of metal rubber, a simple mechanical model was put forward in Ref. [11], as shown in Fig.2. In this model, the hysteretic restoring force of a cycle is decomposed into a nonlinear elastic force and a nonlinear frictional damping force. The large hysteresis loop is expressed as follows:

$$
\left\{\begin{array}{l}
\sigma_{\mathrm{m}}^{\prime}=\sigma_{\mathrm{e}}+\sigma_{\mathrm{f}}=\sigma_{\mathrm{e}}(1+\xi) \\
\sigma_{\mathrm{m}}^{\prime \prime}=\sigma_{\mathrm{e}}-\sigma_{\mathrm{f}}=\sigma_{\mathrm{e}}(1-\xi)
\end{array}\right.
$$

where, $\sigma_{\mathrm{m}}^{\prime}$ is the loading curve, $\sigma_{\mathrm{m}}^{\prime \prime}$ is the unloading curve, $\sigma_{\mathrm{e}}$ is the nonlinear elastic force, $\xi$ is the proportionality constant, and $\sigma_{\mathrm{f}}$ is the nonlinear frictional damping force.

This model is an approximate description of the large hysteresis loop in perfect state, that is, the contact point number of metal rubber is constant, and when the direction of friction force is changed, the entire contact points slide in opposite directions at the same time. It can not reflect the mechanical properties of the large hysteresis loop in the transition phase from loading to unloading, nor can it describe

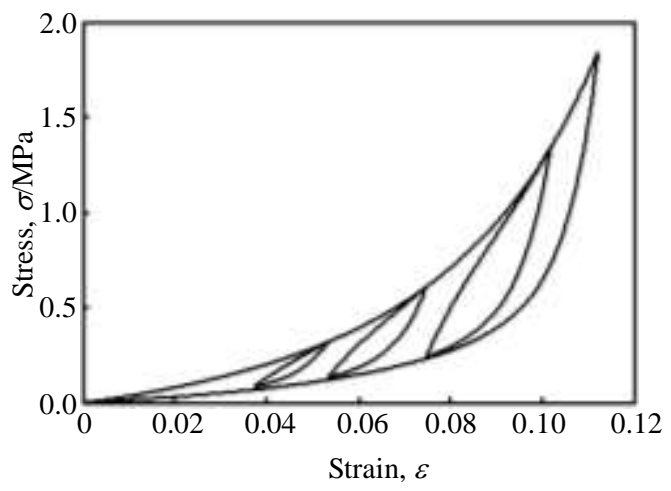

Fig.1 Trial curves of metal rubber

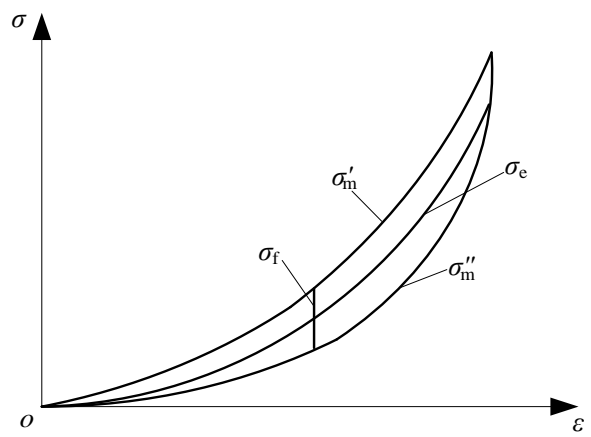

Fig.2 Large hysteresis loop of metal rubber

the fundamental characteristic the friction of which depends on deformation amplitude. Otherwise, it cannot describe the small hysteresis loop in a reasonable manner, neither can predict the small hysteresis loop theoretically. Therefore, in the present paper, based on the simple model above, the hysteresis characteristics of metal rubber will be explored further.

\section{Deformation Analysis of Metal Rubber for Me- soscopic Structure}

\subsection{Mesoscopic deformation model of metal rubber}

According to the preparation technology of metal rubber, in its forming process, because of the extruding and embedding between wire helixes, they are divided into multi-segment curved beams by contact points, as is shown in Fig.3. The curved beams are mutually overlapped and connected together, and the mechanical properties of metal rubber are determined by elastic force of their deformation and interaction force between them ${ }^{[12]}$.

Because it is difficult to accurately ascertain the inner interactive force of metal rubber under the actual conditions, it is necessary to establish a deformation model of its mesoscopic structure, which can accurately simulate the interactive mechanism between curved beams in a certain proportion. Therefore, combined with the mesoscopic deformation mechanism of metal rubber, a mesoscopic deformation model is established, in which a single-turn wire helix is regarded as the mesoscopic structure unit of metal rubber, and according to a certain combination rule, metal rubber is equivalent to a

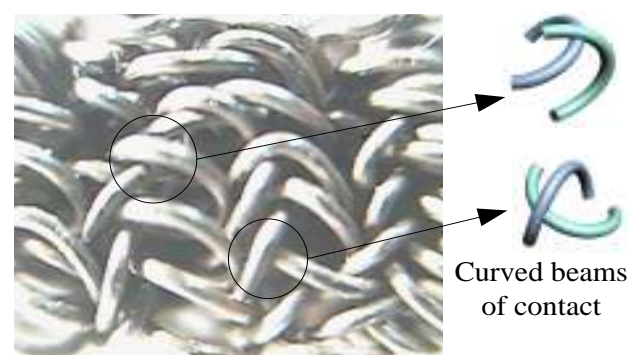

Fig.3 Partial enlarged drawing of metal rubber $(\times 50)$ 
compound of multiple single-turn wire helixes, which are composed of curved beams divided by sliding contact points. Its deformation process under compression is regarded as the process where the number of contact points increases and the length of curved beams decreases gradually. In this model, the length change of curved beams mainly changes the stiffness characteristics of metal rubber, and the number of contact points mainly changes its damping characteristics. In the following analysis, its constitutive model will be established based on the mesoscopic deformation model.

\subsection{Deformation analysis of curved beam}

According to the deformation characteristics of wire helixes and the contact state of curved beams, the influence of spiral angle is ignored, and the curved beam is simplified to the circular arc. In the global coordinate system oxyz and the local coordinate system $o a b c$, the force diagram of the curved beam is established, as is shown in Fig.4. The curved beam with the diameter $D$ is located in the plane $a b$, and the orientation angle is $\alpha$, which is an angle between the plane of the curved beam and the loading direction $z$. Point A of the curved beam is the fixed point, and the contact force $F_{c}$ is applied to point $\mathrm{B}$ along the direction $c$.

Based on the knowledge of structural mechanics ${ }^{[13]}$, the stiffness of the curved beam in direction $c$ is expressed as follows:

$$
\begin{aligned}
& k_{c}=\frac{\pi E d^{4}}{8 D^{3}} \psi(\theta)^{-1} \\
& \psi(\theta)=2 \theta-2 \sin \theta+\left(\frac{3}{2} \theta-2 \sin \theta+\frac{1}{4} \sin 2 \theta\right) v
\end{aligned}
$$

where $(0<\theta<2 \pi)$ is the polar angle of the curved beam, $E$ is the elastic modulus of wire, $v$ is the poison's ratio of wire and $d$ is the diameter of wire.

Through the Matlab software, $\psi(\theta)$ is solved and fitted, and it will be further simplified as:

$$
\psi(\theta)=\frac{1}{\zeta \theta^{-3}+\phi}
$$

where $\zeta$ and $\phi$ are the constants, and their values are related with $v$.

Because the length of the curved beam is generally less than 0.8 loop $^{[14]}$, the influence of $\phi$ is small and can be ignored.

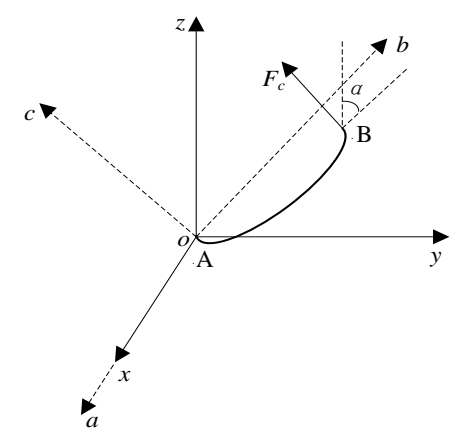

Fig.4 Force diagram of curved beam
Substituting Eq.(3) into Eq.(2), $k_{c}$ is expressed as follows:

$$
k_{c} \approx \frac{\pi \zeta E d^{4}}{8 D^{3}} \theta^{-3}
$$

Substituting the length $l=D \theta / 2$ of the curved beam into Eq.(4), $k_{c}$ is expressed as follows:

$$
k_{c}=\frac{\pi \zeta E d^{4}}{64} l^{-3}
$$

According to the research results in Ref.[15], the model of contact interaction of the curved beam $\mathrm{AB}$ is established at the contact point $\mathrm{B}$, as is shown in Fig.5. The curved beam AB is simplified as the straight beam in the model, and its length is the distance between both ends of the curved beam. The equivalent stiffness and orientation angle $\alpha$ of the straight beam are the same to that of the curved beam, and another curved beam $\mathrm{BC}$ which is contacted with $\mathrm{AB}$ is simplified as the rigid rod in parallel to the loading direction $z$. The straight beam and the rigid rod interact at the contact point $\mathrm{B}$ in the normal direction.

According to Fig.5, the elastic force of the curved beam in the direction $z$ is expressed as follows:

$$
F_{z}=F_{c} \sin \alpha
$$

During deformation process of the curved beam, the contact point moves from point $\mathrm{B}$ to point $\mathrm{B}^{\prime}$, and according to the displacement relationship of $\mathrm{BB}^{\prime}$ and $\mathrm{BB}^{\prime \prime}, F_{c}$ is expressed as follow:

$$
F_{c}=k_{c} \Delta z \sin \alpha
$$

where $\Delta z$ is the displacement of the loading direction $z$.

By Eqs.(6) and (7), the equivalent stiffness of the elastic force of the curved beam in the direction $z$ is expressed as follows:

$$
K_{z}=K_{c} \sin ^{2} \alpha
$$

\subsection{Equivalent stiffness of wire helix}

If the length of the curved beam is $l$, the number of the curved beams contained in single-turn wire helix is defined as follows:

$$
\beta=\pi D / l
$$

Then, the equivalent stiffness of the elastic force of the wire helix in the direction $z$ is expressed as follows:

$$
K_{\mathrm{d}}=\beta k_{z}
$$

During deformation process of metal rubber, the length $l$ of

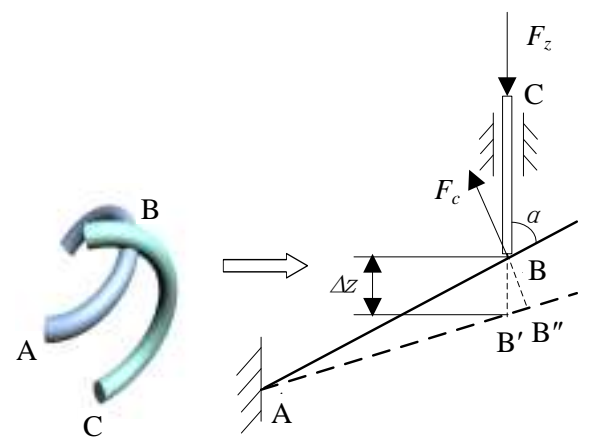

Fig.5 Model of contact interaction of curved beam 
the curved beam decreases with the increase of strain $\varepsilon$, and $l$ is related with $\varepsilon$ linearly ${ }^{[15]} . l$ is expressed as follows:

$$
l=l_{0}(1-b \varepsilon)
$$

where $l_{0}$ is the initial length of the curved beam, and $b$ is the proportional coefficient.

By Eqs.(5) and (8) (11), $k_{\mathrm{d}}$ is expressed as follows:

$$
k_{\mathrm{d}}=\frac{\pi^{2} \zeta E D d^{4} \sin ^{2} \alpha}{64 l_{0}^{4}}(1-b \varepsilon)^{-4}
$$

\section{Stress- Strain Relation of Metal Rubber}

\subsection{Elastic force of metal rubber}

To assess the mechanical properties of metal rubber, a representative unit body is taken out. It is supposed that there are $n$ single-turn wire helix units in per unit area, and $m$ layers wire helix units in per unit length. They are of parallel relationship in each layer and tandem relation between the layers ${ }^{[8]}$. At the strain for $\varepsilon$, the equivalent stiffness of elastic force of metal rubber $k_{\mathrm{e}}$ can be obtained with Eq.(13).

$$
k_{\mathrm{e}}=\frac{n}{m} k_{\mathrm{d}}
$$

If $\rho_{\mathrm{MR}}$ is the density of metal rubber and $\rho$ is the density of wire, the quantity $M_{\mathrm{V}}$ of wire helix in volume $V$ is given by Eq.(14).

$$
M_{\mathrm{V}}=\frac{4 \bar{\rho}_{\mathrm{MR}} V}{\pi^{2} D d^{2}}
$$

where $\bar{\rho}_{\mathrm{MR}}=\rho_{\mathrm{MR}} / \rho$ is the relative density of metal rubber.

By Eq.(14), the quantity of wire helixes in the unit body is given by Eq.(15).

$$
M=\frac{4 \bar{\rho}_{\mathrm{MR}}}{\pi^{2} D d^{2}}
$$

By Eqs.(12), (13), (15) and $l_{0}=\pi \eta D$ ( $\eta$ is for the proportion factor, $\eta>0)$ assumed, $k_{\mathrm{e}}$ is expressed as follows:

$$
k_{\mathrm{e}}=a E\left[\left(\frac{d}{D}\right)^{10} \bar{\rho}_{\mathrm{MR}}\right]^{1 / 3}(1-b \varepsilon)^{-4}
$$

where $a=2^{-16 / 3} \pi^{-8 / 3} \zeta \eta^{-4} \sin ^{2} \alpha$.

Thus the relationship between the elastic stress and the strain of metal rubber is expressed as follows:

$$
\frac{\mathrm{d} \sigma_{\mathrm{e}}}{\mathrm{d} \varepsilon}=a E\left[\left(\frac{d}{D}\right)^{10} \bar{\rho}_{\mathrm{MR}}\right]^{1 / 3}(1-b \varepsilon)^{-4}
$$

The Eq.(17) integrated, the content in the bracket is developed into a multinomial, and meanwhile, the high-order quantity is ignored. The integral constant is determined by $\varepsilon=0$ and $\sigma_{\mathrm{e}}=0$, thus $\sigma_{\mathrm{e}}$ can be expressed as follows:

$$
\sigma_{\mathrm{e}}=a E\left[\left(\frac{d}{D}\right)^{10} \bar{\rho}_{\mathrm{MR}}\right]^{1 / 3}(1-b \varepsilon)^{-3} \varepsilon
$$

\subsection{Hysteretic restoring force of metal rubber}

The hysteretic restoring force of metal rubber contains two parts, that is, the elastic force and the damping force. The damping force of metal rubber is mainly produced from the friction between contact points, and its value is correlated with the number of contact points. In order to grasp the internal damping of metal rubber more accurately, it is necessary to use a quantitative description for internal contact points on physical nature. If the contact points with the friction force is seen as to satisfy Poisson distribution in three-dimensional space, and the angles between wires and vector directions of friction forces are independent and in random ${ }^{[16]}$, and as well as the cyclic process of loading and unloading of metal rubber can be seen as process which can increase the loading phase in optional modes, as is shown in Fig.6, and continuously change the number of contact points, the proportion relations between number of contact points with friction effect and total number of contact points in the initial loading phase $\mathrm{AB}$, unloading phase $\mathrm{BC}$ and repeated loading phase $\mathrm{CD}$ are as follows:

$$
\begin{aligned}
& \delta_{1}=1-\mathrm{e}^{(-1)^{u} \lambda \varepsilon} \\
& \delta_{2}=\delta_{1}\left(\varepsilon_{\mathrm{B}}\right)-2\left[1-\mathrm{e}^{(-1)^{u} \lambda\left(\varepsilon-\varepsilon_{\mathrm{B}}\right)}\right] \\
& \delta_{3}=\delta_{2}\left(\varepsilon_{\mathrm{C}}\right)+2\left[1-\mathrm{e}^{(-1)^{u} \lambda\left(\varepsilon-\varepsilon_{\mathrm{C}}\right)}\right]
\end{aligned}
$$

where $\lambda$ is the proportion coefficient related with the contact points. $u$ is associated with the loading and unloading phases, in the loading phase $u=1$, unloading phase $u=2$.

According to Eqs.(1) and (18) (21), the friction damping force of metal rubber is obtained by Eq.(22).

$$
\sigma_{\mathrm{f}}^{(i)}=\sigma_{\mathrm{e}} \xi \delta_{i}
$$

Based on Eqs.(18) and (22), the hysteretic restoring force of metal rubber in the initial loading, unloading and repeated loading phases can be established by Eq.(23).

$$
\sigma_{i}=\sigma_{\mathrm{e}}+\sigma_{\mathrm{f}}^{(i)}=\sigma_{\mathrm{e}}\left(1+\xi \delta_{i}\right)
$$

where the values of $i=1,2,3$, which represent the initial loading, unloading and repeated loading phases of the curves, respectively.

Eq.(23) is the constitutive model of metal rubber, which can reflect the effects of basic structural parameters such as the diameter $d$, the elastic modulus $E$, the relative density $\bar{\rho}_{\mathrm{MR}}$, and the orientation angle $\alpha$ and so on. In the formula, the parameters $a, b, \xi$ and $\lambda$ can be obtained by the least squares fitting based on the unloading and loading test data of the large hysteresis loop.

\section{Metal Rubber Test and Model Validation}

\subsection{Test equipment and specimens}

Jinan Tianchen WDW-T200 electronic universal testing

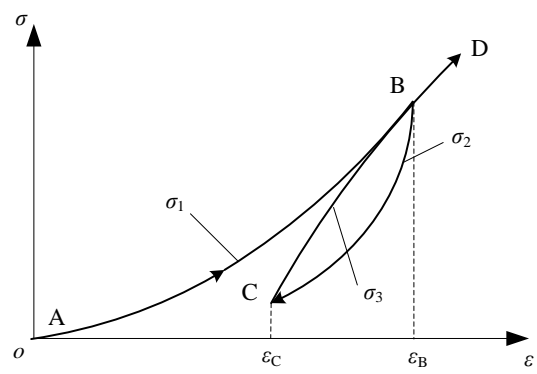

Fig.6 Cyclic process of loading and unloading of metal rubber 
machine (Fig.7a) was used in the present experiment. The austenitic stainless steel wires with the 0Cr18Ni9Ti brand were used as metal rubber specimens, and their parameters were the wire diameter $d=0.3 \mathrm{~mm}$, the density $\rho=7.9 \mathrm{~g} / \mathrm{cm}^{3}$, the elastic modulus $E=210 \mathrm{GPa}$, and the diameter $D=3.2 \mathrm{~mm}$. To verify the model, two metal rubber specimens with different relative densities were made (Fig.7b), and their relative densities are 0.29 and 0.35 .

\subsection{Test and verification}

Substituting the experimental data of the loading and unloading of the large hysteresis loop of metal rubber specimens into the Eq.(23), the parameters $a, b, \xi$ and $\lambda$ are obtained by the least squares fitting. The comparison between the theoretical results and the experimental results is shown in Fig.8.

The curve ADA is the fitting result of the large hysteresis loop, and the curves BFB and CEC are the results of theoretical

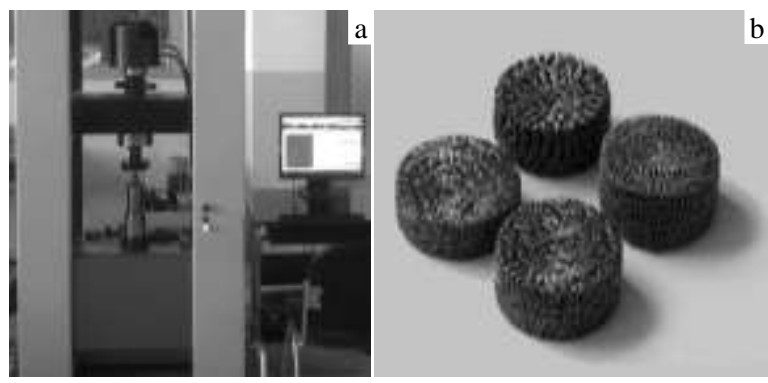

Fig.7 Pictures of testing machine and specimens: (a) testing machine and (b) metal rubber specimens
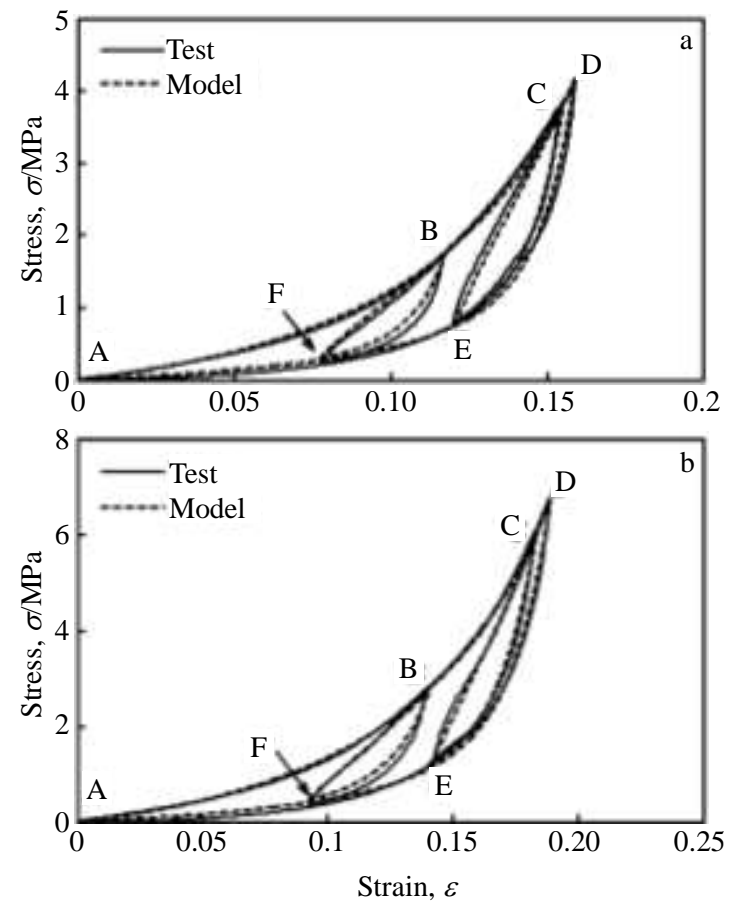

Fig.8 Comparison of theoretical results and experimental results: (a) relative density for 0.29 and (b) relative density for 0.35 prediction of the small hysteresis loops under different predeformations. From the curve ADA, it can be seen that the fitting result of the large hysteresis loop of the model is coincided basically with the experimental data, and it reflects the mechanical properties of the transition phase of the large hysteresis loop perfectly. From the curves BFB and CEC it can be seen the model has a good prediction for the small hysteresis loops, and the model reflects the deformation trend of the small hysteresis loops under different predeformations. The result shows that the theoretical model can describe the hysteresis characteristics of metal rubber. It should be pointed out that the deformation range of metal rubber for the large hysteresis loop should be as broad as possible to expand the fitting range, so as to effectively improve prediction precision of the small hysteresis loops.

\section{Conclusions}

1) The elastic deformation of curved beams and the friction damping between contact points are the essential causes of hysteresis characteristics of metal rubber.

2) The constitutive model of metal rubber can be established based on the hysteresis characteristics. The model contains basic structural parameters of metal rubber, and it is of guiding significance for its design.

3) The experimental results of metal rubber are in conformity with the theoretical results. The model can accurately predict the curves of hysteretic restoring force of metal rubber under different predeformations.

\section{References}

1 Li Yuyan, Huang Xieqing, Mao Wenxiong. Mechanics of Composite Materials[J], 2005, 41: 303

2 Yan Hui, Jiang Hongyuan, Zhao Hongyu et al. Rare Metal Materials and Engineering[J], 2011, 40(12): 2092 (in Chinese)

3 Jiang Hongyuan, Guo Yadong, Chen Zhaobo et al. Rare Metal Materials and Engineering [J], 2009, 38(12): 2116 (in Chinese)

4 Li Shengbo, Yan Hui, Jiang Hongyuan et al. Acta Physica Sinica[J], 2012, 61(1): 010702 (in Chinese)

5 Hao Huirong, Bai Bongbai, Hou Junfang et al. Journal of Vibration and Shock[J], 2008, 27(11): 105 (in Chinese)

6 Li Yuming, Peng Wei, Bai Hongbai et al. Chinese Journal of Mechanical Engineering[J], 2005, 41(9): 38 (in Chinese)

7 Yan Hui, Jiang Hongyuan, Liu Wenjian et al. Acta Physica Sinica $[\mathrm{J}], 2009,58(8): 5238$ (in Chinese)

8 Peng Wei, Bai Hongbai, Zheng Jian et al. Journal of Experimental Mechanics[J], 2005, 20(3): 455 (in Chinese)

9 Li Yuyan, Huang Xieqing. Journal of Aeronautical Materials[J], 2010, 30(2): 94 (in Chinese)

10 Guo Baoting, Zhu Zigen, Cui Rongfan et al. Journal of Aerospace Power[J], 2004, 19(3): 314 (in Chinese)

11 Zhu Bing, Ma Yanhong, Hong Jie. Journal of Beijing University of Aeronautics and Astronautics[J], 2011, 37(10): 1298 (in Chinese) 
12 Zuo H, Chen Y H, Bai H B. Int J Mech Mater Des[J], 2005, 2: 269

13 Long Yuqiu, Bao Shihua. Structural Mechanics[M]. Beijing: Higher Education Press, 2000: 280 (in Chinese)

14 Song Kai. Investigation on Theory and Experiment of Nonlinear Discrete Cell of Metal-rubber[D]. Xi'an: Xi'an Jiaotong University, 2004: 30 (in Chinese)
15 Чегодаев Д Е, Translated by Li Zhongying. The Design of Metallic Rubber Component $[\mathrm{M}]$. Beijing: National Defence Industry Press, 2000: 90 (in Chinese)

16 Ao Hongrui, Jiang Hongyuan, Xia Yuhong et al. Journal of China University of Mining \& Technology[J], 2004, 33(4): 453 (in Chinese)

\section{基于细观力学方法的金属橡胶迟滞特性本构模型}

曹风利, 白鸿柏, 李冬伟, 任国全, 李国璋

(军械工程学院, 河北 石家庄 050003)

摘 要: 依据金属像胶典型细观结构特征, 对金属橡胶压缩变形金属线匝的空间位形和接触模式进行了分析, 揭示了金属橡胶变形的细 观物理机制。提出了基于变长度曲梁的细观结构单元以及曲梁间的接触作用模型, 结合摩擦接触点分布规律, 建立了包含金属丝直径、 金属丝弹性模量、螺旋卷直径、金属橡胶相对密度等基本结构参数的金属橡胶迟滞特性本构模型，实现了金属橡胶初始加载、卸载和重 复加载过程恢复力曲线的描述, 从理论上解释了金属橡胶的弹性性能和多点接触的干摩擦阻尼特性。通过不同相对密度金属橡胶试件对 所建模型进行试验验证, 发现理论预测和试验结果基本一致, 为金属像胶刚度和阻尼的预估以及产品的设计提供了理论依据。

关键词: 金属橡胶; 力学特性; 阻尼材料; 曲线梁

作者简介: 曹风利, 男, 1978 年生, 博士生, 讲师, 军械工程学院车辆与电气工程系, 河北 石家庄 050003, 电话: 0311-87994743, E-mail: zxhpjyscfl@sina.com 